\title{
Association of Serum Soluble CD163 with Polymyositis and Dermatomyositis, Especially in Anti-MDA5 Antibody-positive Cases
}

\author{
Hidenaga Kawasumi, Yasuhiro Katsumata, Akira Nishino, Shinya Hirahara, Yasushi Kawaguchi, \\ Masataka Kuwana, and Hisashi Yamanaka
}

\begin{abstract}
Objective. We elucidated the association of serum soluble CD163 (sCD163) with rapidly progressive interstitial lung disease (RP-ILD), autoantibody profiles, and serum ferritin in patients with polymyositis (PM), classic dermatomyositis (DM), and clinical amyopathic dermatomyositis (CADM). Methods. Serum sCD163 levels were retrospectively measured by ELISA in patients with PM, classic DM, and CADM, as well as in healthy controls (HC). Repeat sera samples were obtained posttreatment from available patients. The associations between serum sCD163 levels and clinical information were analyzed.

Results. Serum sCD163 levels in patients with PM/classic DM/CADM were significantly higher than those in $\mathrm{HC}$ ( $\mathrm{n}=72,56,34$, and 68 , respectively; $\mathrm{p}<0.001$ for all comparisons). No significant difference was observed between serum SCD163 levels in patients with and without ILD $(p=0.16)$ or between those with RP-ILD and chronic ILD ( $\mathrm{p}=0.21)$. Serum sCD163 levels were significantly higher in patients with anti-MDA5 antibodies $(n=27)$ than in those without $(p=0.001)$. Serum sCD163 levels were weakly correlated with serum ferritin levels in the patients with PM, classic DM, and CADM $(r=0.21)$. Serum sCD163 levels decreased significantly following treatment in all patient groups $(\mathrm{p}=0.003)$.

Conclusion. The present results suggest an association of serum sCD163 with PM, classic DM, and CADM, especially in anti-MDA5 antibody-positive cases. However, serum sCD163 levels were not specifically associated with ILD or RP-ILD. (First Release April 15 2018; J Rheumatol 2018;45:947-55; doi:10.3899/jrheum.170997)
\end{abstract}

Key Indexing Terms:

POLYMYOSITIS

DERMATOMYOSITIS

MDA5

INTERSTITIAL LUNG DISEASE

FERRITINS

Polymyositis (PM) and dermatomyositis (DM) are characterized clinically by weakness and low skeletal muscle endurance and histopathologically by the presence of T cells,

From the Institute of Rheumatology, Tokyo Women's Medical University; Department of Allergy and Rheumatology, Nippon Medical School Graduate School of Medicine, Tokyo, Japan.

Supported in part by the Japanese Ministry of Education, Culture, Sports, Science, and Technology Grants-in-Aid for Scientific Research (KAKENHI Grant Number 17K16215). This study was also supported in part by the Nagao Takeshi Nanbyo Foundation. The funders of the study had no role in the study design, conduct of the study, data collection, or data interpretation or preparation of the report. MK holds a patent on an anti-MDA5 antibody measuring kit.

H. Kawasumi, MD, Institute of Rheumatology, Tokyo Women's Medical University; Y. Katsumata, MD, PhD, Institute of Rheumatology, Tokyo Women's Medical University; N. Nishino, MD, Institute of Rheumatology, Tokyo Women's Medical University; S. Hirahara, MD, Institute of Rheumatology, Tokyo Women's Medical University; Y. Kawaguchi, MD, $P h D$, Institute of Rheumatology, Tokyo Women's Medical University; M. Kuwana, MD, PhD, Department of Allergy and Rheumatology, Nippon Medical School Graduate School of Medicine; H. Yamanaka, MD, PhD, Institute of Rheumatology, Tokyo Women's Medical University.

Address correspondence to Dr. Y. Katsumata, Institute of Rheumatology, Tokyo Women's Medical University, 10-22 Kawada-cho, Shinjuku-ku,

Tokyo 162-0054, Japan.E-mail: katsumata@twmu.ac.jp

Accepted for publication January 19, 2018. macrophages, dendritic cells, B cells, and plasma cells in muscle tissue ${ }^{1,2}$. In addition, a subset of patients with DM have cutaneous lesions in the absence of muscle weakness, known as clinically amyopathic dermatomyositis $(\mathrm{CADM})^{2}$. $\mathrm{PM} / \mathrm{DM}$ is often complicated by interstitial lung disease $(\mathrm{ILD})^{3}$. Although therapeutic strategies have improved the clinical courses of patients with PM/DM ${ }^{4,5,6}$, ILD is a major prognostic factor for $\mathrm{PM} / \mathrm{DM}^{7}$. In particular, rapidly progressive (RP)-ILD, mostly complicated with CADM and/or antimelanoma differentiation-associated gene 5 (anti-MDA5) antibodies, is a life-threatening complication ${ }^{8}$.

Predicting disease progression, the prognosis, and treatment response are important when making treatment decisions, given the heterogeneity of the clinical course of $\mathrm{PM} / \mathrm{DM}$-associated ILD ${ }^{9}$. We have reported previously that high levels of serum ferritin, an iron-binding protein, are associated with the severity and prognosis of ILD with $\mathrm{PM} /$ classic DM/CADM, particularly in a patient with anti-MDA5 antibodies ${ }^{8,10,11}$. In addition, we demonstrated that accumulated macrophages synthesize ferritin in the lung and bone marrow of a patient with RP-ILD associated with

Personal non-commercial use only. The Journal of Rheumatology Copyright (c) 2018. All rights reserved. 
$\mathrm{DM}^{12}$. We also reported that proinflammatory cytokines, such as interleukin (IL)-6 and IL-18, are involved in the pathogenesis of RP-ILD ${ }^{12,13,14}$. A series of our previous studies $^{12,13,14}$ led us to conjecture that macrophages are extremely activated in patients with RP-ILD, and that the high levels of serum ferritin are probably produced through activation of macrophages along with a flood of cytokines, similar to patients with macrophage activation syndrome ${ }^{15}$. Although the mechanism of hyperferritinemia in CADM-related and/or anti-MDA5 antibody-related RP-ILD remains elusive, we hypothesized that the high level of serum ferritin might be secreted by systemically activated macrophages. Although the detailed secretory pathway of serum ferritin is not completely understood, macrophages, hepatocytes, and Kupffer cells secrete ferritin ${ }^{15}$. In addition, macrophages are generally considered to be responsible for the production of cytokines and to be of utmost importance in the production and secretion of serum ferritin in 4 uncommon medical conditions characterized by high levels of ferritin, such as macrophage activation syndrome, adult-onset Still disease, catastrophic antiphospholipid syndrome, and septic shock ${ }^{15}$.

CD163 is a glycoprotein expressed by peripheral monocytes and activated macrophages ${ }^{16,17}$. CD163 is shed from activated macrophages during acute or chronic inflammation and is released into peripheral blood in a soluble form $^{18}$. Thus, soluble CD163 (sCD163) in the sera suggests macrophage activation. It has been reported that an increase in serum sCD163 level suggests macrophage activation in some diseases, such as sepsis, lymphoma, and macrophage activation syndrome $\mathrm{e}^{19,20,21}$. In addition, a few studies have suggested an association between sCD163 and PM/DM, particularly in patients with PM/DM-associated ILD, but not with autoantibody profiles ${ }^{22,23}$. However, such an association remains inconclusive because of the statistical methodologies of those studies and the small numbers of cases included. Further, the association between sCD163 and ferritin has scarcely been studied. In our present study, we elucidated the association between serum sCD163 and RP-ILD, autoantibody profiles, and serum ferritin in patients with $\mathrm{PM} /$ classic DM/CADM.

\section{MATERIALS AND METHODS}

Study subjects and sample collection. Adult patients with $\mathrm{PM} / \mathrm{classic}$ DM/CADM and healthy controls (HC) were eligible for our present study. Some patients' serum samples used in our present study were collected previously and stored for unspecified medical studies under general consent from 1994 to 2016, and other samples were prospectively collected specifically for our present study at Tokyo Women's Medical University under written informed consent. Sera from healthy subjects were used as the HC. All sera were stored at $-80^{\circ} \mathrm{C}$ before use. PM/classic DM and CADM were diagnosed according to Bohan and Peter's criteria ${ }^{24,25}$, and Sontheimer's criteria $^{26}$, respectively. Overlapping patients with other collagen tissue diseases and patients complicated with active infection were excluded to avoid potential confounding. All patients with PM/classic DM/CADM were referred to our hospital because of active disease, and all initial serum samples were collected before starting or reinforcing immunosuppressive treatment, usually upon admission. Sera were collected again later from available patients at some time after treatment. The intervals between the time of initial evaluation and reevaluation ranged from 289 to 1658 days (median 619 days). The immunosuppressive treatment was determined according to physician preference in each individual, based on clinically available information and without prior knowledge of the serum sCD163 level. Clinical, radiological, and laboratory data were collected retrospectively from the medical records. ILD was defined as the presence of a diffuse parenchymal lung disease, such as reticular opacity, ground-glass opacity, and honeycombing on high-resolution computed tomography (HRCT). RP-ILD was defined as a rapidly progressive ILD within 3 months of the onset of respiratory symptoms, whereas chronic ILD was defined as progressive dyspnea that developed slowly over $>3$ months ${ }^{8,10}$. Response of ILD to treatment was retrospectively judged using Myositis Intention to Treat Activity Index (MITAX) ${ }^{27}$ by 2 mutually independent rheumatologists (HK and YK). In MITAX, ILD is categorized as worse, the same, or improved when $\geq 2$ of the following were fulfilled: (A) dyspnea or cough because of ILD; (B) parenchymal abnormalities on chest radiograph or HRCT and/or ground-glass-shadowing; and/or (C) 10\% change in total lung capacity/forced volume vital capacity (VC) or $15 \%$ change in DLCO by pulmonary function tests. This study was approved by the Ethical Committee of Tokyo Women's Medical University (registration nos. 3600 and 3733), and the Declaration of Helsinki was followed throughout the study.

Assessment of serum myositis-specific autoantibodies. Myositis-specific autoantibodies (MSA) were assessed as described previously ${ }^{28}$. Briefly, anti-aminoacyl-tRNA synthetase (anti-ARS) antibodies (anti-Jo1, anti-EJ, anti-OJ, anti-PL7, anti-PL12, and anti-KS), as well as anti-signal recognition particle antibodies, were identified by RNA immunoprecipitation assays using unlabeled HeLa cell extracts. Anti-MDA5 antibodies were measured by ELISA. Anti-Mi2, antinuclear matrix protein 2, and antitranscriptional intermediary factor $1-\gamma$ antibodies were identified by protein immunoprecipitation assay using 35S-labeled HeLa cell extracts.

Measurement of serum sCD163 levels. The levels of serum sCD163 were determined by commercial solid phase sandwich ELISA kits, using 96-well microplates precoated with a monoclonal antibody specific to human CD163 as the capture antibody, a monoclonal antibody specific for human CD163 conjugated to horseradish peroxidase as the detection antibody, 3,3',5,5'-tetramethylbenzidine substrate, and recombinant human CD163 as the standard (Human CD163 Quantikine ELISA Kit; R\&D Systems), according to the manufacturer's protocol. The absorbance was measured in a microtiter plate reader (Bio-Rad, Hercules) at a wavelength of $450 \mathrm{~nm}$. Duplicate readings were averaged for each standard, control, and sample, and the average zero standard optical density was subtracted. The best-fit lines were drawn through the standard points using the plot software. The detection range of sCD163 was $1.6 \mathrm{ng} / \mathrm{ml}-100 \mathrm{ng} / \mathrm{ml}$ when samples were not diluted. Samples were diluted 10- or 50-fold. The product datasheet of this ELISA kit gave the mean and range of serum sCD163 levels from apparently healthy volunteers $(\mathrm{n}=36)$ as $472 \mathrm{ng} / \mathrm{ml}$ and 88 to $902 \mathrm{ng} / \mathrm{ml}$, respectively.

Statistical analyses. Two-group comparisons were analyzed using Fisher's exact test for categorical variables and Welch $t$ test for continuous variables. Multiple comparisons for continuous variables were performed using the Tukey-Kramer method. Correlations were evaluated by Pearson correlation coefficient analysis. The difference between the levels of sCD163 before and after treatment was evaluated by the paired $\mathrm{t}$ test. $\mathrm{P}$ values $<0.05$ were considered significant, and all tests were 2-tailed. All statistical analyses were performed using JMP Pro statistical software (version 12.0; SAS Institute) and EZR (Saitama Medical Center, Jichi Medical University, Saitama, Japan $)^{29}$.

\section{RESULTS}

Clinical characteristics of the patients with PM, classic DM, and $C A D M$. Sera were available from 72,56 , and 34 patients

Personal non-commercial use only. The Journal of Rheumatology Copyright $\odot$ (2018. All rights reserved 
with PM, classic DM, and CADM, respectively, and $68 \mathrm{HC}$ and were used in our present study. The median age of these patients was 54 years (range 22-81 yrs). The median disease duration of PM/classic DM/CADM was 7.4 months (range $0.7-233 \mathrm{mos}$ ). The patients and HC were all Japanese. Other clinical characteristics of the patients are summarized in Table 1. Consistent with our previous reports, serum levels of ferritin were significantly higher in patients with RP-ILD than in those with chronic ILD ( $\mathrm{p}=0.04)$.

Serum sCD163 levels in HC. The number of women and men in the $\mathrm{HC}$ were 47 and 21, respectively. The mean age of the healthy controls was 34 years old. There was no significant difference in the sex ratio between the patients with $\mathrm{PM} /$ classic DM/CADM and HC, whereas the patients were older the HC ( $\mathrm{p}=0.32$ and $<0.001$, respectively). Among the $\mathrm{HC}$, there was no significant association between serum sCD163 levels and sex $(\mathrm{p}=0.55)$, and there was no significant correlation between serum sCD163 levels and age $(\mathrm{r}=0.15, \mathrm{p}=0.21)$.

Serum sCD163 levels in patients with PM, classic DM, and $C A D M$. As shown in Figure 1, mean sCD163 levels in patients with PM, classic DM, and CADM, as well as the HC, were $992,867,1061$, and $506 \mathrm{ng} / \mathrm{ml}$, respectively. Serum sCD163 levels in patients with PM, classic DM, and CADM were significantly higher than those in $\mathrm{HC}(\mathrm{p}<0.001$ for all comparisons). However, no significant difference was observed between serum levels of sCD163 in patients with $\mathrm{PM}$, classic DM, and CADM. In addition, no correlation was detected between serum sCD163 levels and serum creatine kinase levels or manual muscle testing scores $(r=0.15$ and -0.08 , respectively), whereas serum levels of sCD163 were

Table 1. Clinical characteristics of the patients with PM, classic DM, and CADM $(n=162)$. Values are $n(\%)$ or median (range) unless otherwise specified.

\begin{tabular}{lc}
\hline Characteristics & Values \\
\hline Age at disease onset, yrs & $54(22-81)$ \\
Sex, women/men & $123(76) / 39(24)$ \\
PM, classic DM, CADM, n & $72,56,34$ \\
Serum CK levels, U/1 & $755(16-12,917)$ \\
Serum ferritin levels, ng/ml & $188(7-8330)$ \\
ILD & $125(77)$ \\
Myositis-specific autoantibodies & \\
$\quad$ Anti-ARS & $55(34)$ \\
Anti-Jo1 & $29(18)$ \\
Anti-MDA5 & $27(17)$ \\
Anti-Mi2 & $4(2)$ \\
Anti-NXP2 & $5(3)$ \\
Anti-SRP & $12(7)$ \\
Anti-TIF1- $\gamma$ & $10(6)$ \\
\hline
\end{tabular}

PM: polymyositis; DM: dermatomyositis; CADM: clinically amyopathic dermatomyositis; CK: creatine kinase; ILD: interstitial lung disease; ARS: aminoacyl-tRNA synthetase; MDA5: melanoma differentiation-associated gene 5; NXP2: nuclear matrix protein-2; SRP: signal recognition particle; TIF1- $\gamma$ : transcriptional intermediary factor $1-\gamma$. weakly correlated with serum aldolase levels in the patients with $\mathrm{PM} /$ classic DM/CADM $(\mathrm{r}=0.25)$. No significant difference in serum sCD163 levels was observed between patients with or without cutaneous ulcers and anti-Ro/SS-A antibodies ( $\mathrm{p}=0.98$ and 0.09 , respectively). Malignancies had been diagnosed in 9 patients within 3 years prior to the diagnoses of PM/classic DM/CADM, whereas malignancies never developed in any patients within 3 years after the diagnoses. There was no significant difference in the serum sCD163 levels between patients with and without malignancies $(\mathrm{p}=0.88)$. There was no significant correlation between serum sCD163 levels and age $(\mathrm{r}=0.04, \mathrm{p}=0.65)$.

Associations between serum sCD163 levels and ILD. As shown in Figure 2A, no significant difference was observed between serum sCD163 levels in patients with and without ILD in the patients with PM/classic DM/CADM ( $p=0.16)$. No significant difference was observed between serum sCD163 levels, even when the patients with ILD were classified into RP-ILD $(\mathrm{n}=36)$ and chronic ILD groups $(\mathrm{n}=89, \mathrm{p}=0.21$; Figure $2 \mathrm{~B})$. There were no significant correlations between serum sCD163 levels and \% VC or $\%$ DLCO $(\mathrm{r}=-0.11$ and $0.02, \mathrm{p}=0.23$ and 0.85 , respectively). Additionally, there was no significant difference in the baseline serum sCD163 levels between patients whose ILD deteriorated later, and those whose ILD was ameliorated later or remained unchanged $(\mathrm{p}=0.71)$. The baseline serum sCD163 levels in the patients who died were not significantly different from the patients who were alive $(\mathrm{p}=0.32)$. By contrast, and consistent with our previous reports $8,10,11,12,13,14$, baseline serum ferritin levels in patients whose ILD deteriorated were significantly higher than those in patients whose ILD was ameliorated or remained unchanged $(\mathrm{p}<0.001)$.

Associations between serum sCD163 levels and myositis-specific autoantibodies. Mean serum sCD163 levels were significantly higher in patients with anti-MDA5 antibodies $(\mathrm{n}=27 ; 1194 \mathrm{ng} / \mathrm{ml})$ than in those without (917 $\mathrm{ng} / \mathrm{ml} ; \mathrm{p}=0.001)$ among the patients with $\mathrm{PM} /$ classic DM/CADM. As shown in Figure 3, when the patients without anti-MDA5 antibodies were further categorized into those with anti-ARS antibodies $(n=55)$ and other MSA $(n=31)$, and the remaining patients (i.e., those without any known MSA; $n=49)$, their mean serum sCD163 levels were 966 $\mathrm{ng} / \mathrm{ml}, 747 \mathrm{ng} / \mathrm{ml}$, and $969 \mathrm{ng} / \mathrm{ml}$, respectively. By multiple comparison analyses, serum sCD163 levels were significantly higher in patients with anti-MDA5 antibodies than in those with other MSA (i.e., non-MDA5/ARS; $\mathrm{p}<0.001)$. Although serum sCD163 levels in the patients with anti-MDA5 tended to be higher than those in patients with anti-ARS and patients without any known MSA, they did not reach statistical significance $(\mathrm{p}=0.10$ and 0.12 , respectively).

Correlations between serum levels of sCD163 and ferritin.

Personal non-commercial use only. The Journal of Rheumatology Copyright @ 2018 . All rights reserved. 


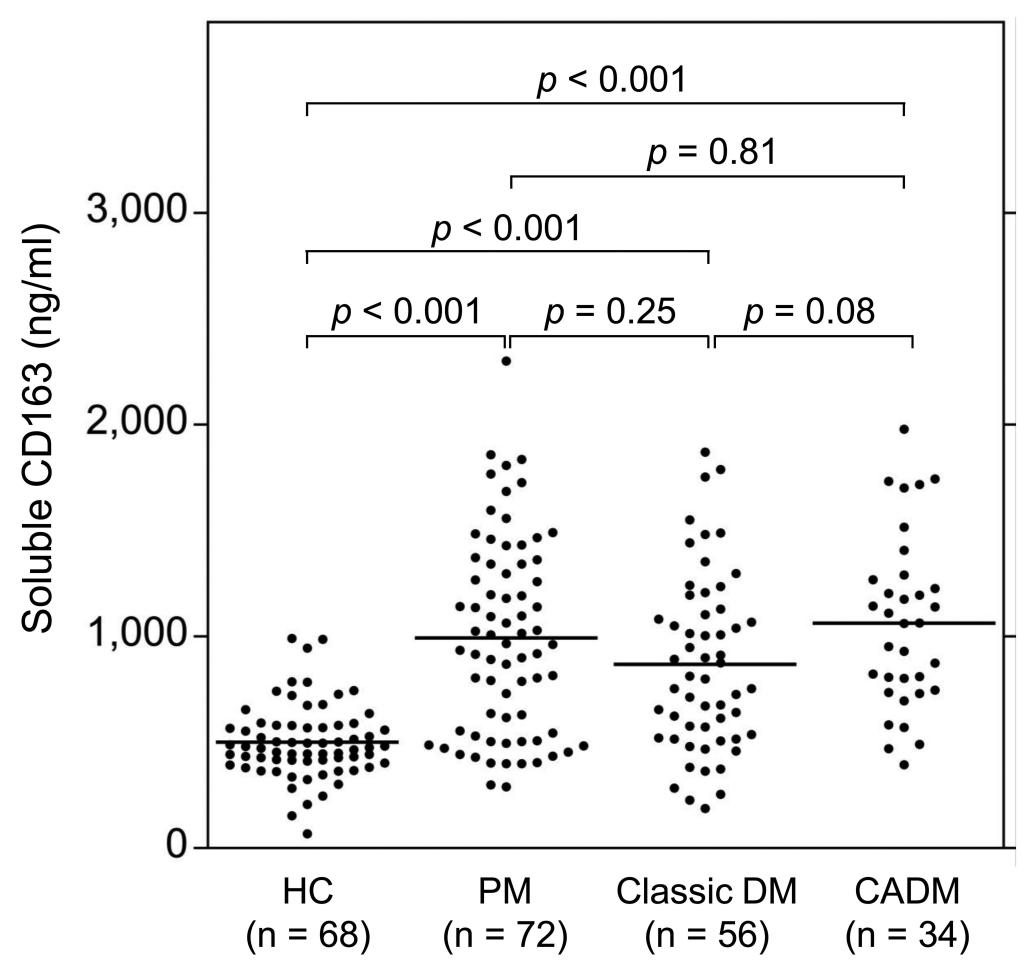

Figure 1. Serum levels of sCD163 in patients with PM, classic DM, and CADM, and HC. Serum sCD163 levels in patients with PM $(n=72)$, classic DM $(n=56)$, and CADM $(n=34)$ were significantly higher than those in $\mathrm{HC}(\mathrm{n}=68$; $\mathrm{p}<0.001$ for all comparisons by the Tukey-Kramer method). Horizontal bars represent the mean levels. sCD163: soluble CD163; PM: polymyositis; DM: dermatomyositis; CADM: clinically amyopathic dermatomyositis; HC: healthy controls.

As shown in Figure 4A, serum sCD163 levels were weakly correlated with serum ferritin levels in the patients with $\mathrm{PM} /$ classic DM/CADM ( $\mathrm{r}=0.21,95 \%$ CI 0 .05-0.36). Serum sCD163 and ferritin levels were moderately correlated in patients with CADM $(r=0.49,95 \%$ CI $0.17-0.71$; Figure 4B). No significant correlation was detected between serum levels of sCD163 and ferritin in patients with RP-ILD $(r=0.10$, Figure $4 C)$. In addition, no significant correlation was observed between serum levels of sCD163 and ferritin in anti-MDA5 antibody-positive patients $(\mathrm{r}=0.04)$.

Serum sCD163 levels before and after treatment in patients with $P M$, classic DM, and CADM. Repeat sera samples were available from 44 patients with PM/classic DM/CADM. As shown in Figure 5, retesting showed that serum sCD163 levels decreased significantly following treatment (median decrease $-26 \%$, range $-67 \%$ to $140 \%, p=0.003$ ). Notably, disease activity of ILD persisted or deteriorated in 7 of the 12 patients $(60 \%)$ whose serum sCD163 levels increased after immunosuppressive treatment.

\section{DISCUSSION}

The major findings of our present study are the following: (1) serum sCD163 was associated with $\mathrm{PM} /$ classic DM/CADM; (2) serum sCD163 levels were significantly higher in patients with anti-MDA5 antibodies than in the remaining patients; (3) serum SCD163 levels were not specifically associated with ILD or RP-ILD; and (4) serum sCD163 levels were only weakly correlated with serum ferritin levels.

Our present study suggests an association between serum sCD163 and PM/classic DM/CADM. The serum levels of sCD163 in patients with PM, classic DM, and CADM were each significantly higher than those in HC. In addition, serum sCD163 levels decreased significantly following treatment. Peng, et al also reported that serum sCD163 levels were significantly elevated and correlated with the Myositis Disease Activity Assessment visual analog scale (VAS) score in patients with $\mathrm{PM} / \mathrm{DM}^{22}$. They also found that serum sCD163 levels decreased significantly after treatment.

For some time, sCD163 has been considered a marker of macrophage activation ${ }^{30}$. Peng, et al reported that patients with high serum sCD163 levels show a higher incidence of CD163+ macrophage infiltration in muscle tissue than do patients with PM/DM and normal sCD163 levels ${ }^{22}$. However, increased sCD163 expression and CD163+ macrophage (M2 macrophage) activation are also closely associated with some other autoimmune diseases, such as rheumatoid arthritis and systemic lupus erythematosus ${ }^{31,32}$. Thus, serum sCD163 alone cannot be used as a disease-specific biomarker. 
A

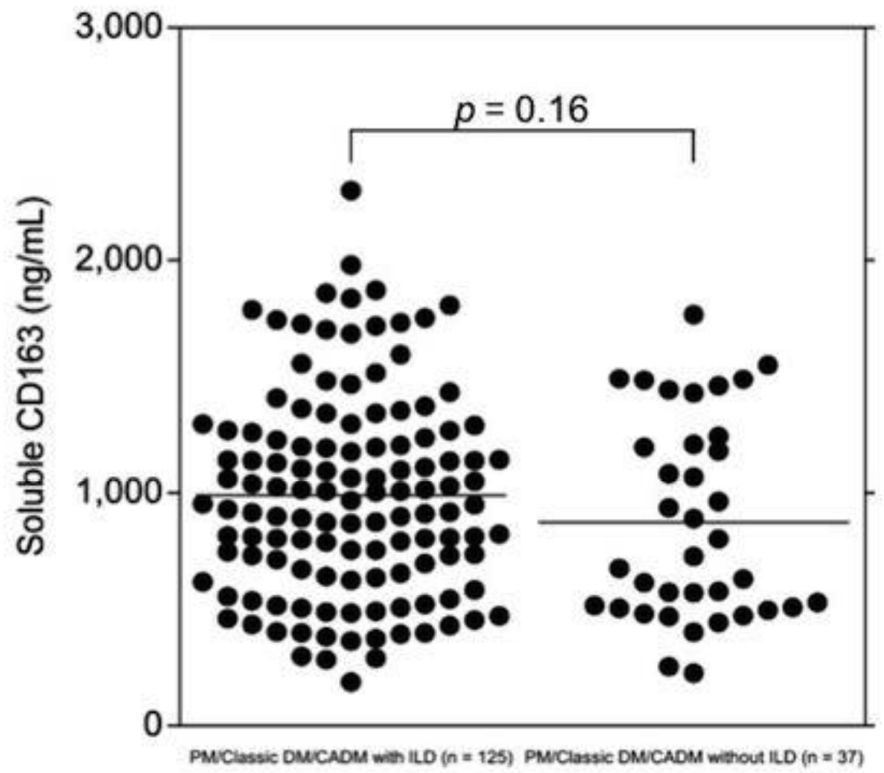

B

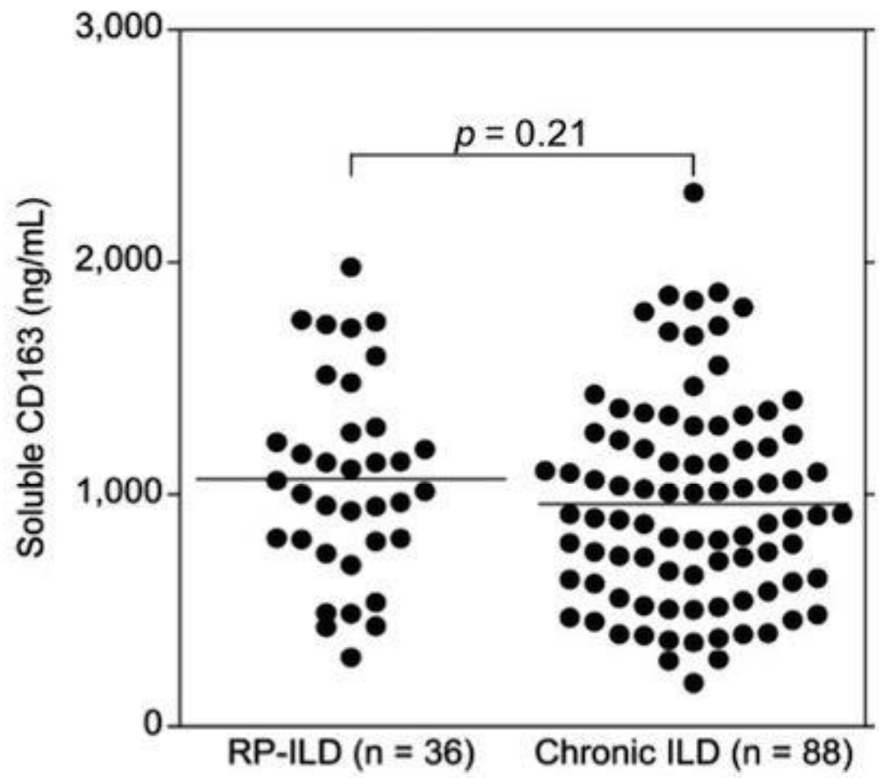

Figure 2. Associations between serum sCD163 levels and ILD. A. No significant difference was observed between serum sCD163 levels in patients with and without ILD ( $\mathrm{n}=125$ and 37 , respectively; $p=0.16$ ). B. No significant difference was observed between serum levels of sCD163, even when the patients with ILD were categorized into those with RP-ILD $(n=36)$ and chronic ILD $(n=89 ; p=0.21)$. P values were determined by Welch $\mathrm{t}$ test. Horizontal bars represent the mean levels. sCD163: soluble CD163; ILD: interstitial lung disease; PM: polymyositis; DM: dermatomyositis; CADM: clinically amyopathic dermatomyositis; ILD: interstitial lung disease; RP-ILD: rapidly progressive ILD.

Serum sCD163 levels were significantly different based on the presence of MSA in our present study. More specifically, serum sCD163 levels were significantly higher in patients with anti-MDA5 antibodies than in those without anti-MDA5 antibodies, especially those with non-MDA5 and non-ARS MSA. MSA and/or their antigen targets may have a role in the pathogenesis of $\mathrm{PM} / \mathrm{DM}^{2}$. For example, the putative role of histidyl-transfer RNA synthetase (Jo1) in bridging innate and adaptive immune responses that ultimately result in myocytotoxicity and lung involvement has been proposed in patients with antisynthetase syndrome $^{33}$. In addition, although the pathogenic roles of

Personal non-commercial use only. The Journal of Rheumatology Copyright (C) 2018. All rights reserved. 


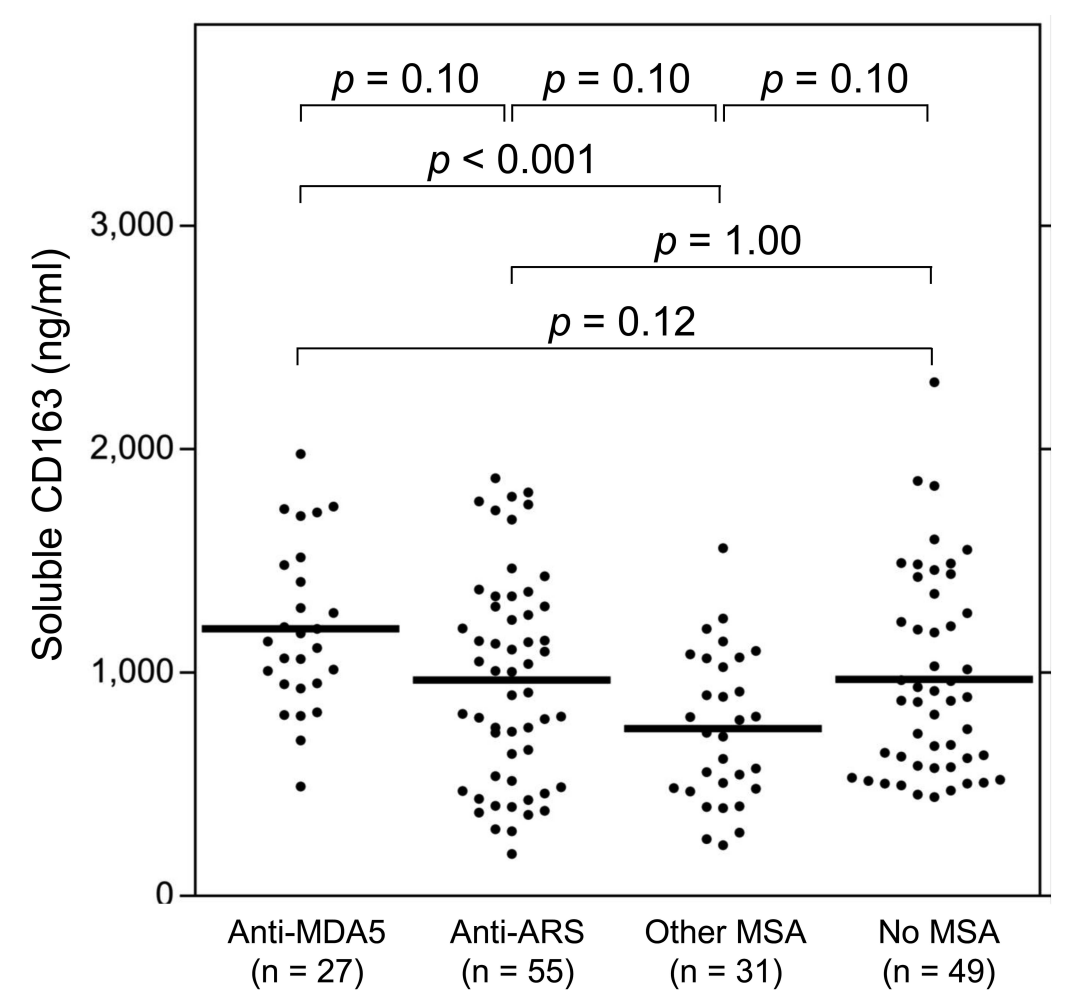

Figure 3. Associations between serum sCD163 levels and MSA. Serum sCD163 levels were significantly higher in patients with anti-MDA5 antibodies than in those with other MSA (i.e., non-MDA5/ARS; $\mathrm{p}<0.001$ ). Although serum sCD163 levels in the patients with anti-MDA5 tended to be higher than those in the patients with anti-ARS and the patients without any known MSA, they did not reach statistical significance ( $\mathrm{p}=0.10$ and 0.12 , respectively). When the patients without anti-MDA5 antibodies were pooled, mean serum sCD163 levels were significantly higher in patients with anti-MDA5 antibodies $(1194 \mathrm{ng} / \mathrm{ml})$ than in those without $(917 \mathrm{ng} / \mathrm{ml} ; \mathrm{p}=0.001)$. P values were determined by the Tukey-Kramer method for multiple comparisons, whereas 2-group comparisons were analyzed using Welch t test. Horizontal bars represent the mean levels. sCD163: soluble CD163; MSA: myositis-specific autoantibodies; MDA5: melanoma differentiation-associated gene 5; ARS: aminoacyl-tRNA synthetase.

anti-MDA5 antibodies are largely unknown, it is speculated that binding of viral dsRNA to MDA5 and the consequent induction of the type I interferon response might be a possible mechanism leading to the autoimmune response ${ }^{34}$. Thus, different levels of association between serum sCD163 levels and the different MSA may be derived from the respective pathomechanisms (such as innate immunity) in each MSA-associated subtype of PM/DM. Somewhat similarly, Enomoto, et al reported that serum sCD163 levels in patients with anti-MDA5-positive PM/DM related-ILD $(n=14)$ was "relatively" higher than in anti-MDA5-negative PM/DM related-ILD patients $(n=34)$, although the result was not statistically significant ${ }^{23}$.

Unexpectedly, serum sCD163 levels were not specifically associated with ILD or RP-ILD in our present study. Additionally, the baseline serum levels of sCD163 in patients whose ILD deteriorated were not significantly different from those in patients whose ILD was ameliorated or remained unchanged. At a glance, these results disagree with those of previous reports. Peng, et al reported that patients with $\mathrm{PM} / \mathrm{DM}$ and ILD have significantly higher serum SCD163 levels than those without ILD ${ }^{22}$. Enomoto, et al reported that serum sCD163 levels in patients with PM/DM related-ILD were "relatively" higher than those in patients with PM/DM without ILD ${ }^{23}$. Peng, et $a l^{22}$ used nonparametric statistics and most of the values in the 2 groups (ILD and non-ILD) were in the same range on the dot plot. As mentioned, elevated sCD163 levels are not disease-specific, and Peng, et al reported that serum sCD163 levels were correlated with global disease activity in patients with $\mathrm{PM} / \mathrm{DM}$ and were associated with muscle macrophage infiltration ${ }^{22}$. Because sCD163 is considered a marker of macrophage activation ${ }^{30}$, we speculated that macrophage activation is involved in both muscle and lung manifestations in PM/classic DM/CADM, and that serum sCD163 levels are eventually elevated in either pathophysiology.

Inconsistently with our initial hypothesis, serum sCD163 levels were only weakly correlated with serum ferritin levels

Personal non-commercial use only. The Journal of Rheumatology Copyright $\subset$ (c) 2018. All rights reserved. 

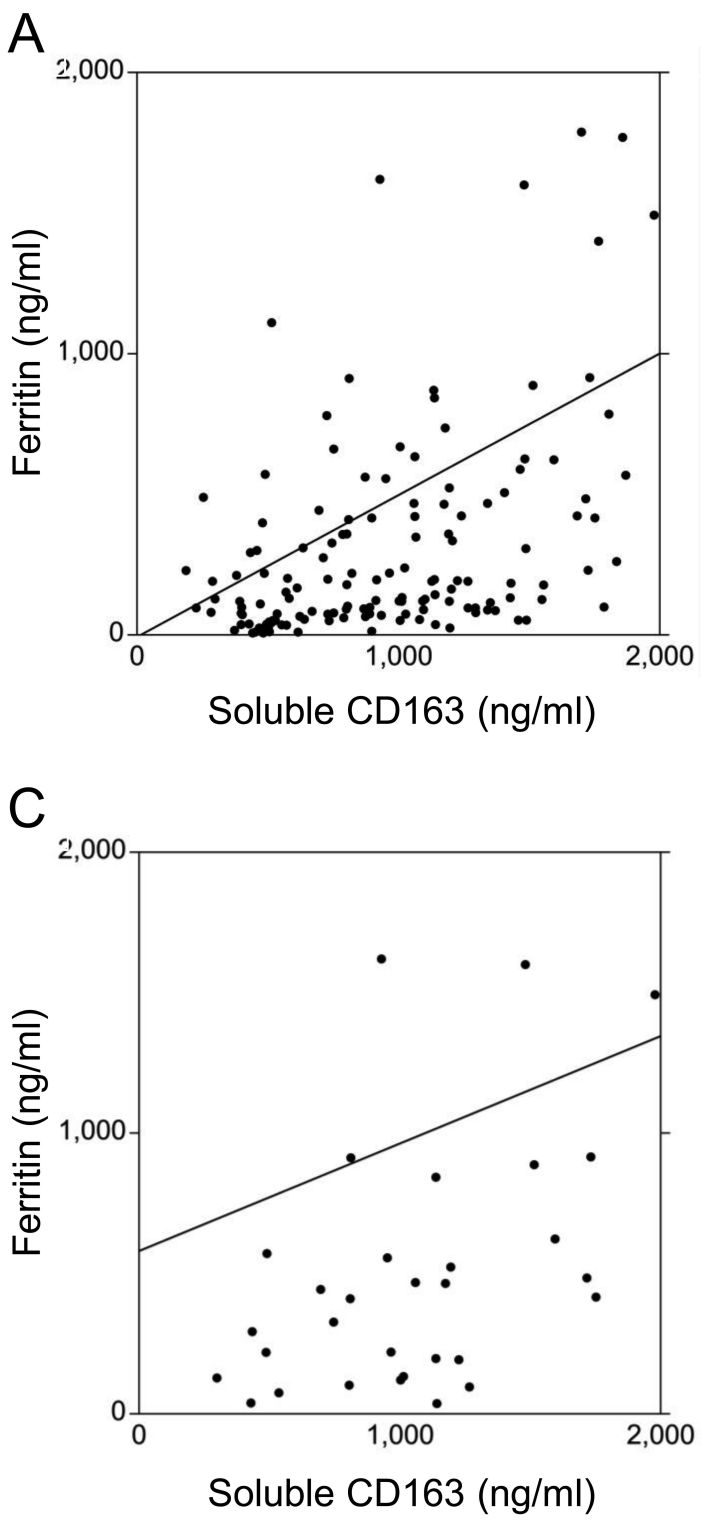

in patients with $\mathrm{PM} /$ classic DM/CADM in our present study. We reported previously that serum IL-6, IL-8, and IL-10 levels are significantly correlated with serum ferritin levels in patients with $\mathrm{PM} / \mathrm{DM}$ and that they are higher in the RP-ILD subset than in the non-ILD subset or the chronic ILD subset $^{14}$. A series of our previous studies ${ }^{10,12,13,14}$ led us to conjecture that the high levels of serum ferritin might be caused by systemically activated macrophages in patients with CADM-related and/or anti-MDA5 antibody-related RP-ILD. However, now we surmise that the hyperferritinemia in this condition is not solely regulated by macrophage activation but that other factors contribute to the phenomenon, although there is some relationship between them, as suggested by the present results. In fact, the expression of ferritin is generally regulated at both the transcriptional and posttranscriptional levels by iron,
B

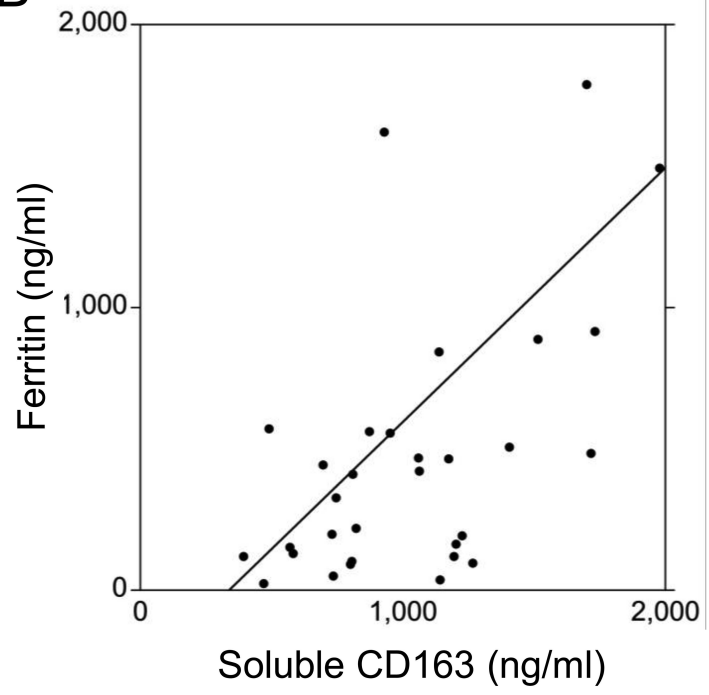

Figure 4. Correlations between serum levels of sCD163 and ferritin. A. Serum levels of sCD163 were weakly correlated with those of ferritin in patients with PM/classic DM/CADM $(r=0.21, p=0.009)$. B. Serum levels of sCD163 and ferritin were moderately correlated when analyzed only among patients with CADM $(r=0.49, \mathrm{p}=$ 0.004). C. No significant correlation was observed between serum levels of SCD163 and ferritin when analyzed only among the patients with RP-ILD $(r=0.10, p=0.54)$. Correlations were evaluated by Pearson correlation coefficient analysis. sCD163: soluble CD163; PM: polymyositis; DM: dermatomyositis; CADM: clinically amyopathic dermatomyositis; RP-ILD: rapidly progressive interstitial lung diseases. cytokine release, chemokine production, lipopolysaccharide, prostaglandins, hormones, growth factors, second messengers, hyperoxia and hypoxia, and oxidative stress ${ }^{15}$. Alternatively, sCD163 may not be ideal for the timely assessment of macrophage activation compared to sCD163 mRNA or CD163 on macrophages.

Several limitations of our present study should be mentioned. First, the validity of our current study could be improved by using a nonretrospective design. For example, the time between the initial and second serum sample collections was too long, and there were not many samples obtained in the short-term after treatment initiation. Second, because the subjects of our present study were all of Japanese ethnicity, it is unclear whether sCD163 has a similar significance in patients of different ethnic backgrounds. Third, we did not examine the presence of CD163+ macrophage infil-

Personal non-commercial use only. The Journal of Rheumatology Copyright @) 2018. All rights reserved. 


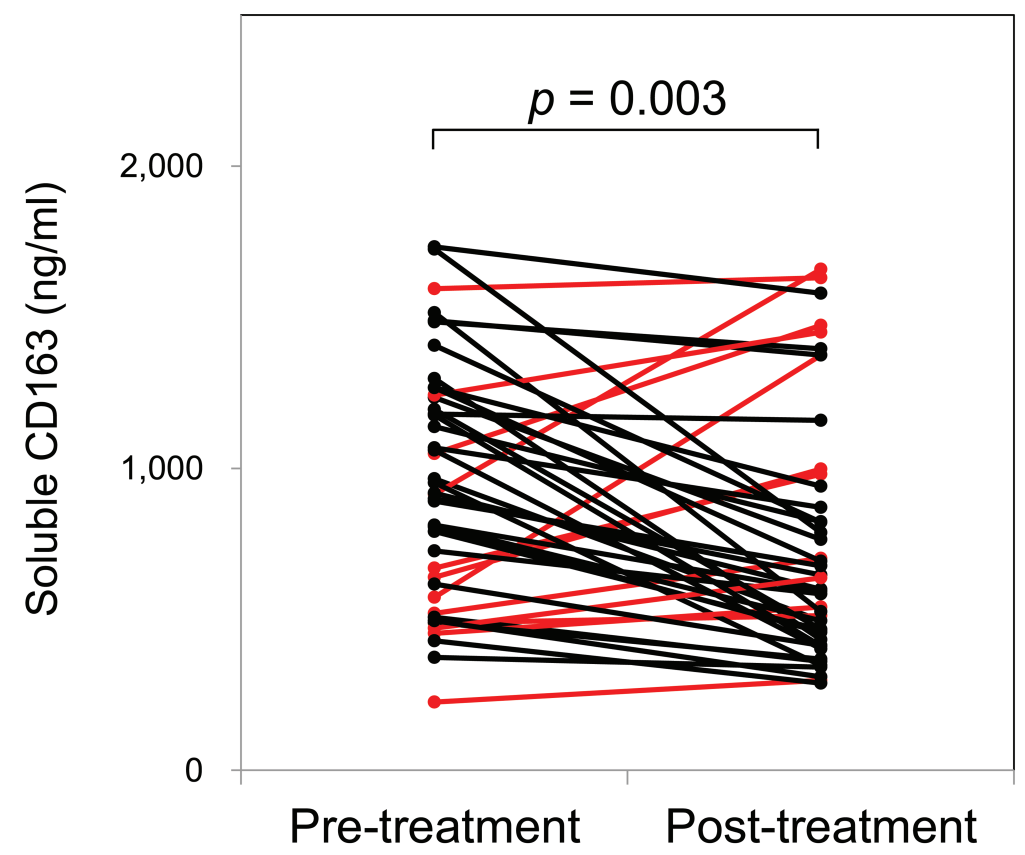

Figure 5. Serum levels of sCD163 before and after treatment in patients with PM, classic DM, and CADM. Serum levels of sCD163 decreased significantly following treatment (median decrease $-26 \%$, range $-67 \%$ to $140 \% ; \mathrm{p}=0.003$ ). $\mathrm{P}$ values were determined by the paired $\mathrm{t}$ test. sCD163 levels increased after treatment and are indicated by red circles and lines. sCD163: soluble CD163; PM: polymyositis; DM: dermatomyositis; CADM: clinically amyopathic dermatomyositis.

tration in muscle or lung tissues. Fourth, the patients were older in age than those of HC. This might seriously affect the results of our present study, although there was no significant correlation between serum sCD163 levels and age among the HC or patients. Finally, we did not use the Myositis Disease Activity Assessment VAS to assess disease activity, although we investigated other factors, such as creatine kinase, aldolase, and manual muscle testing.

Our present study suggests an association between serum sCD163 and PM/classic DM/CADM, especially in anti-MDA5 antibody-positive cases. However, serum sCD163 levels were not specifically associated with ILD or RP-ILD, and the pathomechanisms of how serum ferritin works as a biomarker for CADM-related and/or anti-MDA5 antibody-related RP-ILD remain elusive.

\section{ACKNOWLEDGMENT}

We thank BioMed Proofreading LLC for English-language editing.

\section{REFERENCES}

1. Venalis P, Lundberg IE. Immune mechanisms in polymyositis and dermatomyositis and potential targets for therapy. Rheumatology 2014;53:397-405.

2. Zong M, Lundberg IE. Pathogenesis, classification and treatment of inflammatory myopathies. Nat Rev Rheumatol 2011;7:297-306.

3. Mimori T, Nakashima R, Hosono Y. Interstitial lung disease in myositis: clinical subsets, biomarkers, and treatment. Curr
Rheumatol Rep 2012;14:264-74.

4. Marie I, Hachulla E, Hatron PY, Hellot MF, Levesque H, Devulder $\mathrm{B}$, et al. Polymyositis and dermatomyositis: short term and longterm outcome, and predictive factors of prognosis. J Rheumatol 2001;28:2230-7.

5. Sultan SM, Ioannou Y, Moss K, Isenberg DA. Outcome in patients with idiopathic inflammatory myositis: morbidity and mortality. Rheumatology 2002;41:22-6.

6. Dankó K, Ponyi A, Constantin T, Borgulya G, Szegedi G. Long-term survival of patients with idiopathic inflammatory myopathies according to clinical features: a longitudinal study of 162 cases. Medicine 2004;83:35-42.

7. Marie I. Morbidity and mortality in adult polymyositis and dermatomyositis. Curr Rheumatol Rep 2012;14:275-85.

8. Gono T, Kawaguchi Y, Satoh T, Kuwana M, Katsumata Y, Takagi K, et al. Clinical manifestation and prognostic factor in anti-melanoma differentiation-associated gene 5 antibody-associated interstitial lung disease as a complication of dermatomyositis. Rheumatology 2010;49:1713-9.

9. Gono T, Kuwana M. Inflammatory myopathies: Choosing the right biomarkers to predict ILD in myositis. Nat Rev Rheumatol 2016;12:504-6.

10. Gono T, Kawaguchi Y, Hara M, Masuda I, Katsumata Y, Shinozaki $\mathrm{M}$, et al. Increased ferritin predicts development and severity of acute interstitial lung disease as a complication of dermatomyositis. Rheumatology 2010;49:1354-60.

11. Gono T, Kawaguchi Y, Ozeki E, Ota Y, Satoh T, Kuwana M, et al. Serum ferritin correlates with activity of anti-MDA5 antibody-associated acute interstitial lung disease as a complication of dermatomyositis. Mod Rheumatol 2011;21:223-7. 
12. Gono T, Miyake K, Kawaguchi Y, Kaneko H, Shinozaki M, Yamanaka H. Hyperferritinaemia and macrophage activation in a patient with interstitial lung disease with clinically amyopathic DM Rheumatology 2012;51:1336-8.

13. Gono T, Kaneko H, Kawaguchi Y, Hanaoka M, Kataoka S, Kuwana $\mathrm{M}$, et al. Cytokine profiles in polymyositis and dermatomyositis complicated by rapidly progressive or chronic interstitial lung disease. Rheumatology 2014;53:2196-203.

14. Kawasumi H, Gono T, Kawaguchi Y, Kaneko H, Katsumata Y, Hanaoka M, et al. IL-6, IL-8, and IL-10 are associated with hyperferritinemia in rapidly progressive interstitial lung disease with polymyositis/dermatomyositis. Biomed Res Int 2014;2014:815245.

15. Rosario C, Zandman-Goddard G, Meyron-Holtz EG, D'Cruz DP, Shoenfeld Y. The hyperferritinemic syndrome: macrophage activation syndrome, Still's disease, septic shock and catastrophic antiphospholipid syndrome. BMC Med 2013;11:185.

16. Zwadlo G, Voegeli R, Schulze Osthoff K, Sorg C. A monoclonal antibody to a novel differentiation antigen on human macrophages associated with the down-regulatory phase of the inflammatory process. Exp Cell Biol 1987;55:295-304

17. Radzun HJ, Kreipe H, Bödewadt S, Hansmann ML, Barth J, Parwaresch MR. Ki-M8 monoclonal antibody reactive with an intracytoplasmic antigen of monocyte/macrophage lineage. Blood 1987;69:1320-7.

18. Etzerodt A, Moestrup SK. CD163 and inflammation: biological, diagnostic, and therapeutic aspects. Antioxid Redox Signal 2013;18:2352-63.

19. Weiss M, Schneider EM. Soluble CD163: An age-dependent, anti-inflammatory biomarker predicting outcome in sepsis. Crit Care Med 2006;34:2682-3.

20. Yoon DH, Koh YW, Kang HJ, Kim S, Park CS, Lee SW, et al. CD68 and CD163 as prognostic factors for Korean patients with Hodgkin lymphoma. Eur J Haematol 2012;88:292-305.

21. Coca A, Bundy KW, Marston B, Huggins J, Looney RJ. Macrophage activation syndrome: serological markers and treatment with anti-thymocyte globulin. Clin Immunol 2009;132:10-8.

22. Peng QL, Zhang YL, Shu XM, Yang HB, Zhang L, Chen F, et al. Elevated serum levels of soluble CD163 in polymyositis and dermatomyositis: associated with macrophage infiltration in muscle tissue. J Rheumatol 2015;42:979-87.

23. Enomoto Y, Suzuki Y, Hozumi H, Mori K, Kono M, Karayama M, et al. Clinical significance of soluble CD163 in polymyositis-related or dermatomyositis-related interstitial lung disease. Arthritis Res
Ther 2017;19:9.

24. Bohan A, Peter JB. Polymyositis and dermatomyositis (first of two parts). N Engl J Med 1975;292:344-7.

25. Bohan A, Peter JB. Polymyositis and dermatomyositis (second of two parts). N Engl J Med 1975;292:403-7.

26. Sontheimer RD. Would a new name hasten the acceptance of amyopathic dermatomyositis (dermatomyositis siné myositis) as a distinctive subset within the idiopathic inflammatory dermatomyopathies spectrum of clinical illness? J Am Acad Dermatol 2002;46:626-36.

27. Isenberg DA, Allen E, Farewell V, Ehrenstein MR, Hanna MG, Lundberg IE, et al. International consensus outcome measures for patients with idiopathic inflammatory myopathies. Development and initial validation of myositis activity and damage indices in patients with adult onset disease. Rheumatology 2004;43:49-54

28. Sato S, Hoshino K, Satoh T, Fujita T, Kawakami Y, Fujita T, et al. RNA helicase encoded by melanoma differentiation-associated gene 5 is a major autoantigen in patients with clinically amyopathic dermatomyositis: Association with rapidly progressive interstitial lung disease. Arthritis Rheum 2009;60:2193-200.

29. Kanda Y. Investigation of the freely available easy-to-use software 'EZR' for medical statistics. Bone Marrow Transplant 2013; 48:452-8.

30. Møller HJ, Aerts H, Grønbaek H, Peterslund NA, Hyltoft Petersen P, Hornung N, et al. Soluble CD163: a marker molecule for monocyte/macrophage activity in disease. Scand J Clin Lab Invest Suppl 2002;237:29-33.

31. Zhou D, Wang Y, Chen LU, Zhang W, Luan J. Soluble CD163: a novel biomarker with diagnostic and therapeutic implications in autoimmune diseases. J Rheumatol 2016;43:830

32. Jude C, Dejica D, Samasca G, Balacescu L, Balacescu O. Soluble CD163 serum levels are elevated and correlated with IL-12 and CXCL10 in patients with long-standing rheumatoid arthritis. Rheumatol Int 2013;33:1031-7.

33. Soejima M, Kang EH, Gu X, Katsumata Y, Clemens PR, Ascherman DP. Role of innate immunity in a murine model of histidyl-transfer RNA synthetase (Jo-1)-mediated myositis. Arthritis Rheum 2011;63:479-87.

34. Chen Z, Wang Y, Kuwana M, Xu X, Hu W, Feng X, et al. HLA-DRB1 alleles as genetic risk factors for the development of anti-MDA5 antibodies in patients with dermatomyositis. J Rheumatol 2017;44:1389-93. 